\title{
Erratum
}

\section{Diarylprolinol in the Direct Asymmetric Aldol Reaction of Trifluoromethylacetaldehyde Ethyl Hemiacetal with Aldehyde}

Yujiro Hayashi,* Yusuke Yasui, Tsuyoshi Kawamura, Masahiro Kojima, Hayato Ishikawa Synlett 2011, 485.

In the online and print versions of this article the name of trifluoromethylacetaldehyde ethyl hemiacetal is wrong in the title and through the manuscript. The correct name is trifluoroacetaldehyde ethyl hemiacetal. The authors apologize for this error. 